\title{
A Realization of the q-Deformed Harmonic Oscillator: Rogers-Szegö and Stieltjes-Wigert Polynomials*
}

\author{
Diógenes Galetti \\ Instituto de Física Teórica - IFT \\ Universidade Estadual Paulista - UNESP \\ Rua Pamplona 145, 01405-900, São Paulo, SP, Brazil
}

Received on 31 August, 2002

\begin{abstract}
We discuss some results from $q$-series that can account for the foundations for the introduction of orthogonal polynomials on the circle and on the line, namely the Rogers-Szegö and Stieltjes-Wigert polynomials. These polynomials are explicitly written and their orthogonality is verified. Explicit realizations of the raising and lowering operators for these polynomials are introduced in analogy to those of the Hermite polynomials that are shown to obey the $q$-commutation relations associated with the $q$-deformed harmonic oscillator.
\end{abstract}

\section{Introduction}

The so called $q$-deformed algebras[1, 2, 3, 4] have been object of interest in the physics and mathematical physics literature along the last years, and a great effort has been devoted to its understanding and development $[5,6,7]$. The basic interest in q-deformed algebras resides in the fact that they are deformed versions of the standard Lie algebras, which are recovered as the deformation parameter $q$ goes to unity. And, in this connection, since it is known that the deformed algebras encompass a set of symmetries that is richer than that of the standard Lie algebras, one is tempted to propose that $q$-deformed algebras are the appropriate tool to be used when describing physical systems symmetries which cannot be properly treated within the Lie algebras. Notwithstanding this, the direct interpretation of the deformation parameter in these cases is sometimes incomplete or even completely lacking. To mention some particularly sucessful cases in which the physical meaning of the deformation parameter is clearly established, we could cite for instance, the XXZ-model, where the ferromagnetic/antiferromagnetic nature of a spin $\frac{1}{2}$ chain of length $N$ can be simulated through the introduction of a $q$-deformed algebra[8], or the rotational bands in deformed nuclei and molecules which can be fitted via a $q$-rotor Hamiltonian[9, 10, 11], instead of using the variable moment of inertia (VMI model). In spite of this interpretation difficulty, a solid development has emerged, from the original studies which appeared in connection with problems related to solvable statistical mechanics models [12] and quantum inverse scattering theory[13], which encompass nowadays various branches of mathematical problems related to physical applications, such as deformed superalgebras[14], knot theories[15], noncommutative geometries[16], manybody systems[17](in particular, for nuclear and molecular physics[18]), phase transition[19, 20, 21, 22], $q$-effects in specific heat $[23,24]$, and so on. The introduction of a $q$ deformed bosonic harmonic oscillator is a subject of great interest in this context and, as a tool for providing a boson realization of the quantum algebra $s u_{q}(2)$, brought to light new commutation relations $[25,26,27]$ which have been extensively discussed in the literature.

In this connection, some approaches have been put forward that aim to exhibit realizations of the $q$-deformed harmonic oscillator algebra. The technique using the factorization method $[28,29,30]$ starts from the difference equation associated with the $q$-deformed harmonic oscillator and obtains raising and lowering operators which obey the wellknown $q$-commutation relation. An interesting aspect of this approach is that, associated with that difference equation, there appears some polynomials which can be obtained from the basic hypergeometric functions, and that generalizes the classical polynomials, namely the Jacobi, Laguerre and Hermite polynomials $[31,32,33]$. On the other hand, Feinsilver has discussed how using the three-term recurrence relation for orthogonal polynomials it is possible to obtain a link with raising and lowering operators and discussed their connection with Lie algebras and their $q$-extensions [34, 35]. In the same perspective, the technique using the three-term recurrence relation for the generalized Hermite polynomials was used in [36] to discuss algebraic approach of obtaining sum rules.

In this paper we intend to further follow the technique of obtaining raising and lowering operators from the three-term recurrence relation. We will focus our attention on two $q$ polynomials, namely the Rogers-Szegö and Stieltjes-Wigert polynomials[37, 38], and we will show how it is possible to obtain explicit realizations of the raising and lowering operators associated with these polynomials that are differ-

\footnotetext{
${ }^{*}$ This paper is dedicated to Prof. G.W. Bund, on the occasion of his 70th birthday.
} 
ent from those obtained from the factorization technique and that still satisfy the $q$-commutation relations. The physical motivation for this particular choice of the polynomials is that they both are possible solutions of the $q$-harmonic oscillator and can be used as different bases states for describing physical systems for which the free parameter (the deformation parameter of the algebra) can account for squeezing effects, for instance. Furthermore, the Rogers-Szegö polynomials are orthonormalized on the circle and can therefore be used in connection with angular representations of the harmonic oscillator. In this connection, the Wigner functions for these polynomials have been already obtained and the angle and action marginal distributions calculated[39].

The present paper is organized as follows: In section II we present a brief review of some results from $q$-series that are essential for the discussion of the Rogers-Szegö and Stieltjes-Wigert polynomials, which are presented in Section III. In Section IV we discuss how to obtain raising and lowering operators that satisfy the $q$-commutation relations associated to the $q$-harmonic oscillator. Finally in section V we present our conclusions.

\section{Brief review of some results from q-series}

In order to prepare the scene for the $q$-polynomials we are interested in, let us first introduce some concepts related to the theory of $q$-series[ $6,31,32,40,41]$. The basic notation will be, for $|q|<1$,

such that

$$
(a)_{n} \equiv(a ; q)_{n} \equiv(1-a)(1-a q)\left(1-a q^{2}\right) \ldots\left(1-a q^{n-1}\right)
$$

$$
(a)_{\infty} \equiv(a ; q)_{\infty} \equiv \lim _{n \rightarrow \infty}(a ; q)_{n}
$$

and

$$
(a)_{0} \equiv 1
$$

The particular case should be noted

$$
(q)_{n} \equiv(q ; q)_{n} \equiv(1-q)\left(1-q^{2}\right)\left(1-q^{3}\right) \ldots\left(1-q^{n}\right) .
$$

Furthermore, one can recognize that, for any real $n$, one can write

$$
\begin{gathered}
(a)_{n}=\frac{(a)_{\infty}}{\left(a q^{n}\right)_{\infty}}=\frac{(1-a)(1-a q)\left(1-a q^{2}\right) \ldots\left(1-a q^{n+1}\right) \ldots}{\left(1-a q^{n}\right)\left(1-a q^{n+1}\right) \ldots} \\
=(1-a)(1-a q)\left(1-a q^{2}\right) \ldots\left(1-a q^{n-1}\right) .
\end{gathered}
$$

The Cauchy theorem plays an essential role in this context and states that, for $|t|<1$, the following equality holds

$$
\prod_{n=0}^{\infty} \frac{1-a t q^{n}}{1-t q^{n}}=\sum_{n=0}^{\infty} \frac{(a ; q)_{n}}{(q ; q)_{n}} t^{n}
$$

It is interesting to verify that, starting from the Cauchy theorem, the finite case holds

$$
(x ; q)_{N}=\prod_{s=0}^{N-1}\left(1-q^{s} x\right)=\sum_{s=0}^{N}(-1)^{s}\left[\begin{array}{c}
N \\
s
\end{array}\right]_{q} q^{\frac{s}{2}(s-1)} x^{s},
$$

where

$$
\left[\begin{array}{c}
N \\
s
\end{array}\right]=\frac{(q)_{N}}{(q)_{s}(q)_{N-s}}
$$

is also called $q$-binomial (sometimes it is also called Gauss polynomials [40]).

Hereafter we will use for $n \in N_{0}$ and $q \in[0,1)$

$$
[n]_{q} \equiv[n]:=\frac{1-q^{n}}{1-q}=1+q+\ldots+q^{n-1}
$$

with

$$
[0]=0 \text {, }
$$

which is called a $q$-number(or basic number)[42]. 


\section{III q-Orthogonal Polynomials}

\section{A. Rogers-Szegö polynomials}

The Rogers-Szegö polynomials have been already discussed in the literature $[37,38,40,43]$ so that here we will only recall some important definitions and relations.

The Rogers-Szegö (RS) polynomials are defined as

$$
H_{n}(x ; q)=\sum_{k=0}^{n}\left[\begin{array}{l}
n \\
k
\end{array}\right] x^{k}
$$

and satisfy a three-term recurrence relation

$H_{n+1}(x ; q)=(1+x) H_{n}(x ; q)-\left(1-q^{n}\right) x H_{n-1}(x ; q)$.
Upon recalling that $(q ; q)_{0}=1$, and that the following property holds

$$
\left[\begin{array}{l}
n \\
j
\end{array}\right]=\left[\begin{array}{c}
n \\
n-j
\end{array}\right]
$$

so that

$$
\left[\begin{array}{l}
n \\
0
\end{array}\right]=\left[\begin{array}{l}
n \\
n
\end{array}\right]=1
$$

the first few polynomials can be written, namely

$$
H_{0}(x ; q)=\left[\begin{array}{l}
0 \\
0
\end{array}\right] x^{0}=1
$$

$$
H_{1}(x ; q)=\left[\begin{array}{l}
1 \\
0
\end{array}\right] x^{0}+\left[\begin{array}{l}
1 \\
1
\end{array}\right] x=1+x .
$$

Recalling (2)

$$
H_{2}(x ; q)=1+\frac{(1-q)\left(1-q^{2}\right)}{(1-q)(1-q)} x+x^{2}=1+(1+q) x+x^{2} .
$$

The remaining ones can be directly obtained through the use of the recurrence relation. Now, since we know that in the $q \rightarrow 1$ limit we get back the usual binomial, i.e.,

$$
\lim _{q \rightarrow 1}\left[\begin{array}{l}
n \\
k
\end{array}\right]=\left(\begin{array}{l}
n \\
k
\end{array}\right)
$$

we see that

$$
\lim _{q \rightarrow 1} H_{n}(x ; q)=\lim _{q \rightarrow 1} \sum_{k=0}^{n}\left[\begin{array}{l}
n \\
k
\end{array}\right] x^{k} \rightarrow \sum_{k=0}^{n}\left(\begin{array}{l}
n \\
k
\end{array}\right) x^{k} .
$$

The RS polynomials were shown to be othogonalized on the circle with respect to the Jacobi $\vartheta_{3}(x, t)$ function as the measure[43]. To explicitely show this property, let us consider the proper choice of the variables, namely

$$
H_{n}(x ; q)=H_{n}\left(-q^{-1 / 2} e^{i \varphi} ; q\right) .
$$

Therefore, the orthonormalization condition is written as

$$
I_{m n}=\int_{-\pi}^{\pi} H_{m}\left(-q^{-1 / 2} e^{i \varphi} ; q\right) H_{n}\left(-q^{-1 / 2} e^{-i \varphi} ; q\right) \vartheta_{3}(q, \varphi) \frac{d \varphi}{2 \pi},
$$

with the definition[44, 45]

$$
\vartheta_{3}(q, \varphi)=\sum_{t=-\infty}^{\infty} q^{\frac{t^{2}}{2}} e^{i t \varphi} .
$$

Using the definition of the RS polynomials, (4), we see that, owing to the convergence property of $\vartheta_{3}(q, \varphi)$,

$$
I_{m n}=\sum_{r=0}^{m} \sum_{s=0}^{n}(-1)^{r+s}\left[\begin{array}{l}
m \\
r
\end{array}\right]\left[\begin{array}{l}
n \\
s
\end{array}\right] q^{-\frac{1}{2}(r+s)} \sum_{t=-\infty}^{\infty} q^{\frac{t^{2}}{2}} \delta_{t, s-r},
$$

which gives

$$
I_{m n}=\sum_{r=0}^{m} \sum_{s=0}^{n}(-1)^{r+s}\left[\begin{array}{l}
m \\
r
\end{array}\right]\left[\begin{array}{l}
n \\
s
\end{array}\right] q^{\frac{r}{2(r-1)} q^{\frac{s}{2}(s-1)} q^{-r s} .}
$$

However, this result is precisely that discussed in the Appendix and we directly verify that the orthogonality relation holds

$$
\int_{-\pi}^{\pi} H_{m}\left(-q^{-1 / 2} e^{i \varphi} ; q\right) H_{n}\left(-q^{-1 / 2} e^{-i \varphi} ; q\right) \vartheta_{3}(q, \varphi) \frac{d \varphi}{2 \pi}=\frac{(q ; q)_{n}}{q^{n}} \delta_{m, n} .
$$


From this relation we can extract what is sometimes known as Rogers-Szegö function

$$
R_{n}(\varphi ; q)=\frac{q^{-\frac{n}{2}}}{\left[(q ; q)_{n}\right]^{1 / 2}} H_{n}\left(-q^{-1 / 2} e^{-i \varphi} ; q\right)
$$

In order to see that these polynomials are associated with superpositions of Gaussians on the circle, let us recall the basic relation obeyed by the Jacobi $\vartheta_{3}(q, \varphi)$ function, namely, in general form [44, 45]

$$
\sum_{m=-\infty}^{\infty} \exp \left(-\mu m^{2}-i m \varphi\right)=\sqrt{\frac{\pi}{\mu}} \sum_{n=-\infty}^{\infty} \exp \left[-\frac{1}{4 \mu}(\varphi-2 \pi n)^{2}\right] .
$$

Since we have been using from the beginning

$$
\vartheta_{3}(q, \varphi)=\sum_{t=-\infty}^{\infty} q^{\frac{t^{2}}{2}} e^{i t \varphi}
$$

all we have to do now is to consider $q=\exp (-2 \mu)$ to get expression (6) back. Therefore, the Jacobi $\vartheta_{3}(q, \varphi)$ function is indeed seen to be a superposition of Gaussians and the RS polynomials a family of orthogonalized functions on the circle.

\section{B. Stieltjes-Wigert polynomials}

The Stieltjes-Wigert (SW) polynomials are introduced through their definition [38]

$$
G_{n}(y) \equiv G_{n}(y ; q)=\sum_{r=0}^{n}\left[\begin{array}{l}
n \\
r
\end{array}\right] q^{r(r-n)} y^{r},
$$

and satisfy the three-term recurrence relation

$$
\begin{gathered}
G_{n+1}(y ; q)=(1+y) G_{n}(y ; q) \\
+q^{-n}\left(1-q^{n}\right) y G_{n-1}(y ; q) .
\end{gathered}
$$

The first polynomials are

$$
G_{0}(y ; q)=1
$$

$$
\begin{gathered}
G_{1}(y ; q)=1+y \\
G_{2}(y ; q)=1+\frac{(1+q)}{q} y+y^{2},
\end{gathered}
$$

and the others can be directly obtained from the recurrence relation.

If we specialize the parameters appearing in their definition, we can obtain a family of polynomials that are orthogonal on the line, namely let us consider $y=-q^{n+1 / 2} x$. In this way the SW polynomials read

$$
G_{n}\left(-q^{n+1 / 2} x\right)=\sum_{r=0}^{n}(-1)^{r}\left[\begin{array}{l}
n \\
r
\end{array}\right] q^{r(r+1 / 2)} x^{r}
$$

Let us consider further

$$
q=\exp \left(-\frac{1}{2 k^{2}}\right)
$$

and

$$
x=\exp \left(\frac{u}{k^{2}}\right)
$$

With these choices we have

$$
G_{n}(u ; q)=\sum_{r=0}^{n}(-1)^{r}\left[\begin{array}{l}
n \\
r
\end{array}\right] \exp \left[-\frac{r}{2 k^{2}}(r+1 / 2)\right] \exp \left(\frac{u r}{k^{2}}\right) .
$$

To show that these polynomials are orthogonal on the line we have to verify that a weight function $p(x)$ exists such that

$$
\int_{0}^{\infty} G_{m}\left(-q^{m+1 / 2} x\right) G_{n}\left(-q^{n+1 / 2} x\right) p(x) d x=F(q, n) \delta_{n, m}
$$

where $F(q, n)$ is some definite function of $q$ and $n$. In fact it has been shown[46] that

$$
p(x)=\frac{k}{\pi^{1 / 2}} \exp \left(-k^{2} \log ^{2} x\right)
$$

is the desired function with

$$
2 k^{2}=-\frac{1}{\log q}
$$


which is equivalent to expression (8). With the definition (9) we see that

$$
\frac{u^{2}}{k^{2}}=k^{2} \log ^{2} x
$$

so that

$$
p(u)=\frac{k}{\pi^{1 / 2}} \exp \left(-\frac{u^{2}}{k^{2}}\right)
$$

We can now rewrite the modified SW polynomials in such a form to embody the weight function, namely,

$$
\begin{gathered}
S_{n}(u ; q)=p^{1 / 2}(u) G_{n}(u ; q) \\
=\frac{k^{1 / 2}}{\pi^{1 / 4}} \exp \left(-\frac{u^{2}}{2 k^{2}}\right) \sum_{r=0}^{n}(-1)^{r}\left[\begin{array}{l}
n \\
r
\end{array}\right] \exp \left[-\frac{r}{2 k^{2}}(r+1 / 2)\right] \exp \left(\frac{u r}{k^{2}}\right) \\
=\frac{k^{1 / 2}}{\pi^{1 / 4}} \sum_{r=0}^{n}(-1)^{r}\left[\begin{array}{l}
n \\
r
\end{array}\right] \exp \left[-\frac{1}{2 k^{2}}(u-r)^{2}\right] \exp \left(-\frac{r}{4 k^{2}}\right) .
\end{gathered}
$$

It is worth noticing that each of these polynomials can be written as a superposition of $n$ Gaussians centered at the points $r$. Furthermore these polynomials are orthogonal on the line, since now $-\infty \leq u \leq \infty$, and, in particular, are orthogonal to a single Gaussian centered around the origin since this corresponds to $S_{0}(u ; q)$. In order to see this property let us consider

$$
\mathcal{I}_{m n}=\int_{-\infty}^{\infty} S_{m}(u ; q) S_{n}(u ; q) \frac{\exp \left(\frac{u}{k^{2}}\right)}{k^{2}} d u
$$

Substituting the explicit expressions for the polynomials, Eq.(10)

$$
\begin{gathered}
\mathcal{I}_{m n}=\frac{1}{k \pi^{1 / 2}} \sum_{r, s=0}^{m, n}(-1)^{r+s}\left[\begin{array}{c}
n \\
r
\end{array}\right]\left[\begin{array}{c}
m \\
s
\end{array}\right] \exp \left(-\frac{r^{2}}{2 k^{2}}-\frac{r}{4 k^{2}}-\frac{s^{2}}{2 k^{2}}-\frac{s}{4 k^{2}}\right) \\
\times \int_{-\infty}^{\infty} \exp \left[-\frac{u^{2}}{k^{2}}+\frac{u}{k^{2}}(r+s+1)\right] d u
\end{gathered}
$$

which gives

$$
\mathcal{I}_{m n}=\exp \left(\frac{1}{4 k^{2}}\right) \sum_{r, s=0}^{m, n}(-1)^{r+s}\left[\begin{array}{l}
n \\
r
\end{array}\right]\left[\begin{array}{c}
m \\
s
\end{array}\right] \exp \left[-\frac{r(r-1)}{4 k^{2}}\right] \exp \left[-\frac{s(s-1)}{4 k^{2}}\right] \exp \left(\frac{r s}{2 k^{2}}\right) .
$$

Recalling that we are considering $q=\exp \left(-\frac{1}{2 k^{2}}\right)$, we end up with

$$
\begin{gathered}
\mathcal{I}_{m n}=q^{-1 / 2} \sum_{r, s=0}^{m, n}(-1)^{r+s}\left[\begin{array}{c}
n \\
r
\end{array}\right]\left[\begin{array}{c}
m \\
s
\end{array}\right] q^{-\frac{r}{2}(r-1)} q^{-\frac{s}{2}(s-1)} q^{-r s} \\
=q^{-1 / 2} I_{m n}=q^{-1 / 2} q^{-n}(q ; q)_{n} \delta_{n, m},
\end{gathered}
$$

which proves the orthogonality of the SW polynomials.

In this form we have shown that this particular kind of Gaussian superpositions, which correspond to the StieltjesWigert polynomials, can be used as a orthogonal set of states on the line.

\section{IV q- Orthogonal Polynomials and q- Algebras}

\section{A. Hermite polynomials}

Before we treat the connection between these $q$ polynomials and the realization of the deformed harmonic oscillator algebra we are interested in, let us recall the properties of a classical polynomial[47], namely, the Hermite polynomial. 
As is well known from the theory of classical polynomials, the Hermite polynomials obey a three-term recurrence relation

$$
H_{n+1}(x)=2 x H_{n}(x)-2 n H_{n-1}(x)
$$

as well as a differentiation relation

$$
\frac{d}{d x} H_{n}(x)=2 n H_{n-1}(x) .
$$

If we insert (12) in (11), we get

$$
H_{n+1}(x)=\left(2 x-\frac{d}{d x}\right) H_{n}(x)
$$

which points to the introduction of a raising operator[36], defined as

$$
R=2 x-\frac{d}{d x}
$$

such that the set of Hermite polynomials can be generated by the application of this operator to the first polynomial, viz., $H_{0}(x)=1$,

$$
R^{n} H_{0}(x)=H_{n}(x) .
$$

In exactly the same form we can define a lowering operator directly from Eq.(12), namely

$$
\frac{1}{2} \frac{d}{d x} H_{n}(x)=L H_{n}(x)=n H_{n-1}(x) .
$$

It is a direct task to verify that these operators satisfy the canonical commutation relation

$$
[L, R]=1 \text {. }
$$

In this form, from Eqs.(11) and (12) we were able to write a pair of operators that satisfy a characteristic canonical commutation relation, although they are not the usual creation and annihilation operators associated to the quantum mechanics harmonic oscillator. Furthermore, we can also construct a number operator in the form

$$
N=R L
$$

such that

$$
N H_{n}(x)=R L H_{n}(x)=n H_{n}(x) .
$$

Using the explicit representation of those operators we have

$$
\left[\left(2 x-\frac{d}{d x}\right)\left(\frac{1}{2} \frac{d}{d x}\right)-n\right] H_{n}(x)=0,
$$

which can be cast in the standard form of a second order differential equation for the Hermite polynomials

$$
\left(\frac{d^{2}}{d x^{2}}-2 x \frac{d}{d x}+2 n\right) H_{n}(x)=0 .
$$

It can also be directly verified that the operators $N, R$ and $L$ obey the standard commutation relations

$$
[N, R]=R
$$

and

$$
[N, L]=-L \text {. }
$$

In this form, we see that we can obtain a raising, a lowering and a number operator from the two basic relations satisfied by the Hermite polynomials, namely, the three-term recurrence relation and the differentiation relation respectively, such that they satisfy the well known commutation relations.

On the other hand, let us consider the usual Hilbert space $\mathcal{H}$ which is spanned by the vectors $|n\rangle$, generated from the vacuum $|0\rangle$ by the raising operator $R$. Together with the lowering operator $L$, the following relations hold

$$
\begin{gathered}
L R-R L=1, \\
\langle 0 \mid 0\rangle=1, \\
|n\rangle=R^{n}|0\rangle, \\
L|0\rangle=0 .
\end{gathered}
$$

In particular, the following can also be proven by the use of the previous relations

$$
\begin{gathered}
R|n\rangle=|n+1\rangle, \\
L|n\rangle=n|n-1\rangle, \\
\langle m \mid n\rangle=n ! \delta_{m n} .
\end{gathered}
$$

Now, it is immediately seen that the raising and lowering operators obtained from the basic properties of the Hermite polynomials are explicit realizations of the above particular algebra (it is worth noticing that it is not the usual harmonic oscillator algebra). This clearly points then to the fact that the three-term recurrence relation together with the differentiation relation of the Hermite polynomials give an way of constructing that particular realization of an algebra closely related to the harmonic oscillator one.

\section{B. Rogers-Szegö polynomials}

As already pointed out by some authors[27, 28, 29], it is possible to introduce a $q$-generalization of the harmonic oscillator algebra by considering a Hilbert space $\mathcal{H}_{q}$, where $q$ is the parameter already discussed before, spanned by the vectors $|n\rangle$, which are generated from the vaccum $|0\rangle$ by the action of a raising operator $R$. In a similar way the relations hold

$$
L R-q R L=1,
$$

where $q$ is a real parameter, $0<q<1$, or equivalently, as presented by Feinsilver[35],

$$
L R-R L=q^{N},
$$

where $N$ is the usual number operator, 


$$
\begin{gathered}
\langle 0 \mid 0\rangle=1, \\
|n\rangle=R^{n}|0\rangle, \\
L|0\rangle=0 .
\end{gathered}
$$

In a similar way as before, we can also verify that the relations follow

$$
\begin{gathered}
R|n\rangle=|n+1\rangle, \\
L|n\rangle=[n]|n-1\rangle, \\
\langle m \mid n\rangle=[n] ! \delta_{m n},
\end{gathered}
$$

where the $q$-number factorial is given by

$$
[n] !=[1][2][3] \ldots[n] .
$$

It is then seen that the vectors $([n] !)^{-1 / 2}|n\rangle$ form an orthonormal basis set and this Hilbert space $\mathcal{H}_{q}$ consists of all vectors $|u\rangle=\sum_{n=0}^{\infty} u_{n}|n\rangle$, with complex $u_{n}$ such that $\langle u \mid u\rangle=\sum_{n=0}^{\infty}\left|u_{n}\right|^{2}[n]_{q}$ ! is finite.

Guided by the results of the previous section, and the analogy between the Hermite polynomials and the RogersSzegö polynomials, as emphasized by Carlitz[37, 38], in this section we want to obtain the corresponding raising, lowering and $q$-number operators and to look for the corresponding commutation relations.

As already shown, Eq.(5),

$$
H_{n+1}(x, q)=(1+x) H_{n}(x ; q)-\left(1-q^{n}\right) x H_{n-1}(x, q) \text {. }
$$

Now we will be interested in a simple relation, namely the analogous expression to the differentiation relation of the Hermite polynomials, Eq.(12). In fact, the RS polynomials do not exhibit such a relation, but if we calculate the $q$ difference of these polynomials, designated by the operator $\Delta$, i.e.,

$$
\Delta H_{n}(x ; q)=H_{n}(x ; q)-H_{n}(x q ; q),
$$

by the direct use of Eq.(4) we get

$$
\Delta H_{n}(x ; q)=\left(1-q^{n}\right) x H_{n-1}(x ; q),
$$

which establishes, together with the recurrence relation, Eq.(5), a pair of equations relating neighbouring polynomials. In the same form we pointed out for the Hermite polynomials, if we substitute the above expression for $H_{n-1}(x ; q)$ in Eq.(5), we end up with

$$
H_{n+1}(x, q)=[(1+x)-\Delta] H_{n}(x ; q),
$$

which can again be understood as the action of a raising operator for the RS polynomials, namely

$$
A_{+} H_{n}(x ; q)=H_{n+1}(x, q),
$$

where

$$
A_{+}=(1+x)-\Delta .
$$

Exactly in the same form we can identify a lowering operator directly from Eq.(14), viz.,

$$
A_{-}=\frac{1}{x} \Delta
$$

such that

$$
A_{-} H_{n}(x ; q)=\left(1-q^{n}\right) H_{n-1}(x ; q),
$$

and verify that the operator defined as $A_{+} A_{-}$gives

$$
A_{+} A_{-} H_{n}(x ; q)=\left(1-q^{n}\right) H_{n}(x ; q)
$$

whose eigenvalues are in close connection with the $q$ number, Eq.(3). Hence, guided by these results, we redefine these operators by the introduction of the Jackson's $q$ derivative[31, 32], namely

$$
D_{q} \phi(x)=\frac{\phi(x)-\phi(q x)}{x(1-q)}=\frac{\Delta \phi(x)}{x(1-q)},
$$

which goes to the usual derivative in the $q \rightarrow 1$ limit. With this expression, the raising and lowering operators may be written as

$$
S_{+}=(1+x)-(1-q) x D_{q}
$$

and

$$
S_{-}=D_{q}
$$

respectively. The $S_{+}$operator acts on the RS polynomials in the same form as $A_{+}$, while now

$$
S_{-} H_{n}(x ; q)=[n] H_{n-1}(x ; q)
$$

so that, calling $N_{q}=S_{+} S_{-}$, we see that

$$
N_{q} H_{n}(x ; q)=[n] H_{n}(x ; q)=\frac{1-q^{n}}{1-q} H_{n}(x ; q),
$$

which plays the role of the $q$-number operator.

The commutation relation between these operators can be directly obtained giving

$$
\left[S_{-}, S_{+}\right]=1-(1-q) N_{q} .
$$

On the other hand, since we know Eq.(15), we further observe that, making use of the standard number operator $N$

$$
N H_{n}(x ; q)=n H_{n}(x ; q),
$$

we may write

$$
N_{q}=\frac{1-q^{N}}{1-q}=[N]
$$

such that

$$
\left[S_{-}, S_{+}\right]=q^{N},
$$


which is the particular case of the commutation relation for the $q$-deformed harmonic oscillator we have presented above. In the same form we can also obtain

$$
\begin{gathered}
{\left[N_{q}, S_{-}\right]=-q^{N} S_{-},} \\
{\left[N_{q}, S_{+}\right]=S_{+} q^{N},}
\end{gathered}
$$

while

$$
\left[N, S_{-}\right]=-S_{-}
$$

and

$$
\left[N, S_{+}\right]=S_{+} \text {. }
$$

It is also immediate to see that the $q$-commutation relation of these operators is given by

$$
S_{-} S_{+}-q S_{+} S_{-}=[N+1]-q[N]=1,
$$

which is the equivalent form of the commutation relation of the $q$-deformed harmonic oscillator as also stated above, Eq.(13).

Now, from Eq.(15), and using the explicit realization of the raising and lowering operators, we can write

$$
N_{q} H_{n}(x ; q)=\left[(1+x) D_{q}-(1-q) x D_{q}^{2}\right] H_{n}(x ; q)=[n] H_{n}(x ; q)
$$

from which we get the second order $q$-differential equation obeyed by the Rogers-Szegö polynomials

$$
\left[x D_{q}^{2}-\frac{(1+x)}{(1-q)} D_{q}+\frac{[n]}{(1-q)}\right] H_{n}(x ; q)=0 .
$$

\section{Stieltjes-Wigert polynomials}

The Stieltjes-Wigert polynomials satisfy the three-term recurrence relation, Eq.(7)

$G_{n+1}(x ; q)=(1+x) G_{n}(x ; q)+q^{-n}\left(1-q^{n}\right) x G_{n-1}(x ; q)$,

as already stated. Instead of deducing the raising and lowering operators in this case from the very beginning, we shall take advantage of an important property shown by Carlitz[37], namely that the SW polynomials are directly related to the RS polynomials through the transformation

$$
G_{n}(x ; q)=H_{n}\left(x ; q^{-1}\right) .
$$

This means that properties related to the RS polynomials can be put in direct correspondence with properties of the SW polynomials so that we refrain from explicitely deducing all them here again. Instead, using this connection, we can look for the raising and lowering operators associated to these polynomials taking advantage of the ones already obtained in the previous section. Therefore, knowing that

$$
S_{+}=(1+x)-(1-q) x D_{q}
$$

and

$$
S_{-}=D_{q}
$$

we propose the new operators

$$
W_{+}=(1+x)-\left(1-q^{-1}\right) x D_{q^{-1}},
$$

and

$$
W_{-}=D_{q^{-1}}
$$

as the desired operators associated to the SW polynomials. It is direct to see that they act on those polynomials as

$$
W_{+} G_{n}(x ; q)=G_{n+1}(x ; q)
$$

and

$W_{-} G_{n}(x ; q)=\frac{1-q^{-n}}{1-q^{-1}} G_{n-1}(x ; q)=[n]_{q^{-1}} G_{n-1}(x ; q)$

respectively.

As in the case of the RS polynomials, we can obtain the commutation relation between these operators, namely

$$
\left[W_{-}, W_{+}\right]=1-\left(1-q^{-1}\right) W_{+} W_{-}
$$

which can also be rewritten in terms of the standard number operator as

$$
\left[W_{-}, W_{+}\right]=q^{-N}
$$

and it is also direct to see that the $q$-commutation relation obeyed by these operators is

$$
W_{-} W_{+}-q^{-1} W_{+} W_{-}=1
$$

as expected. 


\section{Conclusions}

In this paper we have discussed two $q$-polynomials presented in the mathematical literature, namely the RogersSzegö and Stieltjes-Wigert polynomials and presented some interesting properties they exhibit in connection with the classical Hermite polynomials. Upon recalling a technique of constructing explicit realizations of raising and lowering operators which satisfy an algebra akin to the usual harmonic oscillator algebra, through the use of the three-term recurrence relation and the differentiation expression of Hermite polynomials, we have shown that a similar procedure can be carried out in the case of those $q$-polynomials. In fact, the Jackson's $q$-derivative replaces the usual one and the procedure we have proposed here was shown to give origin to new realizations of the $q$-deformed algebra associated to the $q$-deformed harmonic oscillator, which then obey the well-known commutation and $q$-commutation relations presented in the literature.

In what refers to the Stieltjes-Wigert polynomials it is to be observed that raising and lowering operators - obeying the $q$-deformed harmonic oscillator algebra - have been already obtained for them in a somewhat different approach in Ref.[29]. There the authors factorize the difference equation satisfied by those polynomials and obtain the raising and lowering operators in terms of $q$-difference operators which are still another different realization of the corresponding algebra.

One of the interesting points to be discussed in a future publication in connection with these realizations of the $q$-deformed harmonic oscillator algebra is that the RogersSzegö polynomials were shown to be orthogonalized on the unit circle, allowing them to be good representations of angle states in the description of the radiation field, in which the deformation parameter of the algebra plays the role of a squeezing parameter. Work along this line is in progress and will be presented in another publication.

\section{Acknowledgement}

We wish to thank Prof. B.M. Pimentel for a critical reading of the manuscript and valuable suggestions. This work was partially supported by Conselho Nacional de Desenvolvimento Científico e Tecnológico, CNPq.

\section{Appendix A: An important equality}

Following Carlitz[38], let us first consider

$$
I_{m n}=\sum_{r=0}^{m} \sum_{s=0}^{n}(-1)^{r+s}\left[\begin{array}{l}
m \\
r
\end{array}\right]\left[\begin{array}{l}
n \\
s
\end{array}\right] q^{-\frac{r}{2}(r-1)} q^{-\frac{s}{2}(s-1)} q^{-r s}
$$

and let us show that $I_{m n}=q^{-n}(q ; q)_{n} \delta_{n m}$. To this end we recall the result already presented in the text, Eq.(1)

$$
(a ; q)_{N}=\prod_{j=0}^{N-1}\left(1-q^{j} a\right)=\sum_{j=0}^{N}(-1)^{j}\left[\begin{array}{c}
N \\
j
\end{array}\right] q^{\frac{1}{2} j(j-1)} a^{j}
$$

and put it in (16) so that

$$
I_{m n}=\sum_{r=0}^{m}(-1)^{r}\left[\begin{array}{c}
m \\
r
\end{array}\right] q^{-\frac{r}{2}(r-1)} \prod_{s=0}^{n-1}\left(1-q^{s-r}\right) .
$$

Now, without any loss of generality, we can assume that $m \leq n$ (the inverse could also be considered). There are two situations to be discussed. First: For $m<n$, it is evident that the product on the rhs of (17) will vanish for all $r$ (the rhs is constituted of a sum of products. Each summand has a product of terms where one of them will give $\left(1-q^{r-r}\right)=0$, since, as $m<n$, $s$ will necessarily assume the value $r$ ). Therefore, the sum only have vanishing summands, since there will always be a zero factor in the products.

Thus

$$
I_{m n}=0 \text { for } m<n .
$$

Second: For $m=n$ there will be only one term to be considered, namely $r=m$, what will give

$$
I_{n n}=(-1)^{n} q^{-\frac{n}{2}(n-1)} \prod_{s=0}^{n-1}\left(1-q^{s-n}\right) .
$$

To calculate this expression, let us explicitely write the product

$$
\begin{gathered}
I_{n n}=(-1)^{n} q^{-\frac{n}{2}(n-1)}\left(1-\frac{1}{q^{n}}\right)\left(1-\frac{1}{q^{n-1}}\right) \ldots\left(1-\frac{1}{q}\right) \\
=(-1)^{n} q^{-\frac{n}{2}(n-1)} \frac{(-1)^{n}\left(1-q^{n}\right)\left(1-q^{n-1}\right) \ldots(1-q)}{q^{n} q^{\frac{n}{2}(n-1)}} \\
=\frac{\left(1-q^{n}\right)\left(1-q^{n-1}\right) \ldots(1-q)}{q^{n}}
\end{gathered}
$$

which, upon identifying the numerator, gives

$$
I_{n n}=\frac{(q ; q)_{n}}{q^{n}} .
$$

This contribution together with (18) gives the final result

$$
I_{m n}=q^{-n}(q ; q)_{n} \delta_{n m} .
$$

\section{References}

[1] V.G. Drinfeld, Proceedings of the International Congress on Mathematics, Vol. 1 (University of California Press, Berkeley, Cal., 1987), p. 798

[2] M. Jimbo, Lett. Math. Phys. 11, 247 (1986).

[3] P. Kulish and N. Reshetikhin, J. Sov. Math. 23, 2435 (1983).

[4] E.K. Sklyanin, Funct. Anal. Appl. 16, 262 (1982).

[5] V. Chari and A. Pressley, A Guide to Quantum Groups (Cambridge University Press, 1995). 
[6] L.C. Biedenharn and M.A. Lohe, Quantum Group Symmetry and Q-Tensor Algebras (World Scientific, Singapore, 1995).

[7] M. Chaichian and A. Demichev, Introduction to Quantum Groups (World Scientific, Singapore, 1996).

[8] V. Pasquier and H. Saleur, Nucl. Phys. B330, 523 (1990).

[9] S. Iwao, Prog. Theor. Phys. 83, 363 (1990).

[10] P.P. Raychev, R.P. Roussev and Yu.F. Smirnov, J. Phys. G16, L137 (1990).

[11] E. Celeghini, R. Giachetti, E. Sorace and M. Tarlini, Phys. Lett. B280, 180 (1992).

[12] R.J. Baxter, Exactly Solved Models in Statistical Mechanics (Academic Press, London, 1982).

[13] E. Sklyanin, L.Takhatajan and L. Fadeev, Theor. Math. Phys. 40, 194 (1979)

[14] M. Chaichian and P. Kulish, Phys. Lett. B234, 72 (1990).

[15] L. Kauffman, Int. J. Mod. Phys. A5, 93 (1990).

[16] Y. Manin, Quantum Groups and Non-Commutative Geometry, (Centre des Recherches Mathématiques, Montreal University Press, Montreal, 1988); A. Connes, Géometry NonCommutative (Intereditions, Paris, 1990).

[17] D. Galetti, J.T. Lunardi, B.M. Pimentel and C.L. Lima, $Q-$ deformed algebras and many-body systems, in Topics in Theoretical Physics, Festschrift for Paulo Leal Ferreira, Eds. V.C. Aguilera-Navarro, D. Galetti, B.M. Pimentel and L. Tomio (Ed. IFT, SP, Brazil, 1995).

[18] D. Bonatsos and C. Daskaloyannis, Prog. Part. Nucl. Phys. 43, 537 (1999)

[19] D. Galetti and B.M. Pimentel, An. Acad. Bras. Ci. 67, 7 (1995); S.S. Avancini, A. Eiras, D. Galetti, B.M. Pimentel and C.L. Lima, J. Phys. A: Math. Gen. 28, 4915 (1995); D. Galetti, B.M. Pimentel, C.L. Lima and J.T. Lunardi, Physica A242, 501 (1997).

[20] S. S. Avancini and J. C. Brunelli, Phys. Lett. A174, 358 (1993).

[21] S.S. Avancini, D.P.Menezes, M.M.W. de Moraes and F.F. de Souza Cruz, J. Phys. A: Math. Gen. 27, 831 (1994).

[22] S.S. Avancini, F.F. de Souza Cruz, D.P. Menezes and M.M.W. de Moraes, J. Phys. A28, 701 (1995).

[23] S. Zhang, Phys. Lett. A202, 18 (1995).

[24] M. R-Monteiro, L. M. C. S. Rodrigues and S. Wulck, Phys. Rev. Lett. 76, 1098 (1996).

[25] L.C. Biedenharn, J. Phys. A22, L873 (1989).

[26] A.J. Macfarlane, J. Phys. A22, 4581 (1989).

[27] M. Arik and D. Coon, J. Math. Phys. 17, 524 (1975).
[28] N.M. Atakishiev and S.K. Suslov, Theor. Math. Phys. 85, 1055 (1991).

[29] N.M. Atakishiev and S.K. Suslov, Theor. Math. Phys. 87, 442 (1991).

[30] N.M. Atakishiev, A. Frank and K.B Wolf, J. Math. Phys. 35, 3253 (1994).

[31] G. Gasper and M. Rahman, Basic Hypergeometric Series in Encyclopedia of Mathematics and its Applications (Cambridge University Press, Cambridge, UK, 1997).

[32] N. Ja. Vilenkin and A.U. Klimyk, Representation of Lie Groups and Special Functions, vol. 3 (Kluwer Academic Publishers, Dordrecht, The Netherlands, 1992).

[33] T.S. Chihara, An Introduction to Orthogonal Polynomials (Gordon and Breach, N.Y., 1978).

[34] P. Feinsilver, Acta Appl. Math. 13, 291 (1988).

[35] P. Feinsilver, Acta Appl. Math. 19, 207 (1990).

[36] J. Daboul and S.S. Mizrahi, Jour. of Group Theory in Phys. 2, 161 (1994)

[37] L. Carlitz, Ann. Math. Pura Applic. 41, 359 (1956).

[38] L. Carlitz, Publicationes Mathematicae 5, 222 (1958).

[39] D. Galetti, S.S. Mizrahi and M. Ruzzi, Proceedings of the VII International Wigner Symposium, Maryland, USA, August 2001 .

[40] G. E. Andrews, The Theory of Partitions in Encyclopedia of Mathematics and its Applications (Addison-Wesley Publ. Co., Reading, Ma, USA, 1976).

[41] G. E. Andrews, q-Series: Their development and application in analysis, number theory, combinatorics, physics, and computer algebra, Regional Conference Series in Mathematics, vol. 26 (American Mathematical Society, Providence, USA, 1986).

[42] F.H. Jackson, Q. J. Pure, Appl. Math. 41, 193 (1910).

[43] G. Szegö, Orthogonal Polynomials, Colloquium Publications, vol. 23 (Americam Mathematical Society, Providence, RI, USA, 1991).

[44] E.T. Whittaker and G.N. Watson, A Course of Modern Analysis (Cambridge University Press, USA, 1969).

[45] R. Bellman, A Brief Introduction to Theta Functions (Holt, Rinehart and Winston, NY, 1961).

[46] S. Wigert, Arkiv för Mat. 27, no.18 (1923).

[47] A. Érdelyi et al. (eds), Higher Transcendental Functions (California Institute of Technology H. Bateman M.S. Project), Vol.2, McGraw Hill, New York (1953). 